\title{
Calcium pyrophosphatase dihydrate (CPPD) crystal deposition disease in a teaching hospital in Kuwait
}

A N Malaviya, I M Al-Shari, A R Al-Shayeb, D Shehab, M A S Hussain, M Al-Mutairy, O M Roberts, S Al-Ghuriear

Abstract

Objective-A Medline electronic search showed a paucity of reports on calcium pyrophosphate dihydrate crystal deposition disease (CPPD-CDD) from the Gulf region. To date only a single case report has been published from this region. Therefore, this study aimed, firstly, at finding out the prevalence of chondrocalfinding out the prevalence of chondrocal-
cinosis in adult Arabs in Kuwait presentcinosis in adult Arabs in Kuwait presenting with knee arthritis and, secondly, at CPPD-CDD among Arabs in Kuwait.

Methods-For the study of the prevalence of chondrocalcinosis 100 consecutive adult patients presenting with knee arthritis were radiologically examined. For the observational study the clinical, laboratory, and radiological findings were analysed in patients with CPPD-CDD seen over a period of five years.

Results-This study showed the presence of chondrocalcinosis in two (2\%) of the 100 adult Kuwaiti and other Middle-Eastern Arab patients $(70$ men, 30 women, median age 50 (range 45-80)) who presented to the rheumatology clinic for the evaluation of knee pain. When the younger age of the group (only three patients aged $>70$ ) is taken into account the figure was comparable with that reported from Western countries. Over a period of five years a total of 2726 new patients were evaluated at the rheumatology clinic of this institution. A diagnosis of crystal arthritis was made in 85 patients $(3 \%)$. Fourteen of these 85 (that is, $16.5 \%$, but $0.5 \%$ of the total cases) were diagnosed with definite (eight patients) or probable (six patients) CPPD-CDD. Different clinical presentations, including that of acute monarthritis (that is, pseudogout), premature generalised osteoarthritis, and polyarticular rheumatoid-like presentations, were seen in different patients. Overlap with true gout, with the additional presence of monosodium urate crystals in the joint aspirate, was seen in two patients.

Conclusion-The present report shows that CPPD-CDD may not be uncommon that CPPD-CDD may not be uncommon degree of clinical awareness and routine examination of joint aspirates with careful analysis for crystals may make it a more common diagnosis in this part of the world. In this regard it is ir note that cases and case series including familial cases have been reported from North Africa, especially Tunisia, indicating that the disease has been well described in Arabs of other geographical regions.

(Ann Rheum Dis 2001;60:416-419)

Calcium pyrophosphate dihydrate crystal deposition disease (CPPD-CDD) is the second most common form of the crystal deposition disease. ${ }^{1}$ However, because of the marked heterogeneity in clinical syndromes associated with CPPD-CDD, its prevalence and incidence are difficult to define. ${ }^{1}$ Most epidemioogical studies are small and have relied upon necropsies or radiological findings of chondrocalcinosis. $^{2}$

A Medline search and search of reviews showed a paucity of reports on CPPD-CDD from developing countries. Only one centre from Tunisia has reported cases and families of CPPD-CDD (cited by Reginato and Hamza $e t$ $\left.a l^{4}\right)$. In addition to reports from this centre, only one series from Thailand and one case report from Qatar in the Gulf region has been o earlie reports from this institution some interesting observations relating to hyperuricaemia and gout were highlighted. ${ }^{7}$ This report describes our experience with CPPD-CDD in a large teaching hospital in Kuwait.

Subjects and methods

The study included a prospective radiologica survey of the knee joints in 100 consecutive patients presenting to the rheumatology service of this institution with knee arthritis during 1997. In addition, the study also included all CPPD-CDD by the rheumatology service of this institution over a period of five years (middle of May 1994 to the middle of 1999)

The diagnostic criteria of Ryan and Mc$\mathrm{Carty}^{8}$ were used for diagnosing definite and probable CPPD-CDD

A standard technique of polarised light microscopy was used to show the presence of crystals in synovial fluid, ${ }^{9}$ modified by using a kit for producing polarised light (for the identification of gout or pseudogout crystal manufactured by M/S Crystal Diagnostics, Division of J \& G Manufacturing Co, Midlothian, Virginia, USA). To improve the sensitivity of the technique lightly centrifuged samples were copy. ${ }^{9}$ 
Results

The 100 consecutive patients presenting with knee pain comprised 70 men and 30 women, with a median age of 50 (range 45-80). The age distribution of the patients was as follows: 39 patients aged $45-49,44$ aged 50-59, 12 aged $60-69,2$ aged $70-79$, and 1 aged $>80$. All the patients were of Middle-Eastern Arab stock, the majority being Kuwaiti (82). Two male Kuwaiti patients aged 50 and 75 , respectively, were found to have chondrocalcinosis in the knee.

Of a total of 2726 patients seen by the rheumatology service over the period of the study, 85 had crystal arthritis - 69 had gout, 14 had CPPD-CDD, and there were two others.
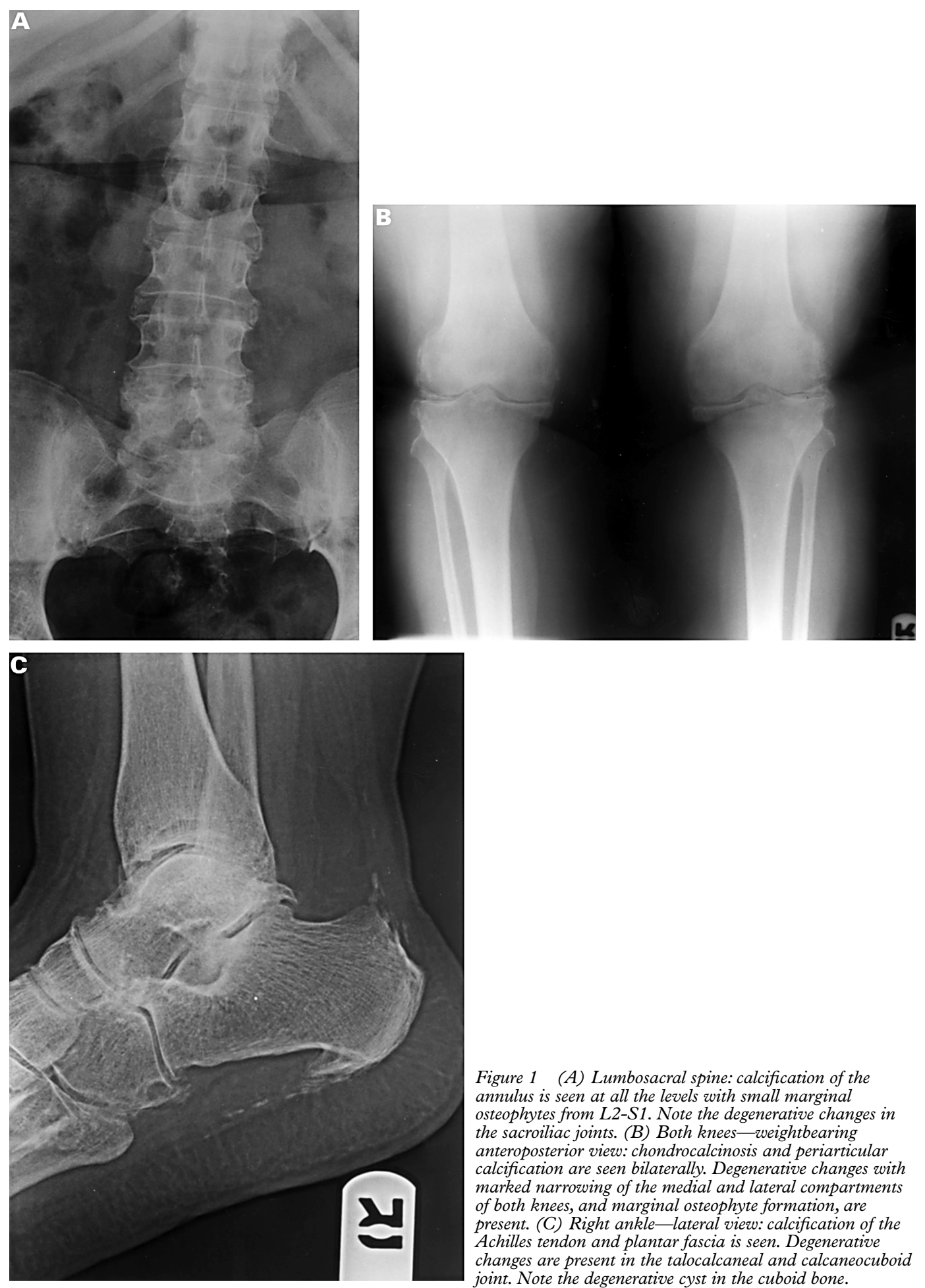

Figure 1 (A) Lumbosacral spine: calcification of the annulus is seen at all the levels with small marginal osteophytes from L2-S1. Note the degenerative changes in the sacroiliac joints. (B) Both knees - weightbearing chesterior view. chondrocalcinosis and periarticular. marked narrozing of the medial and lateral compartments of both knees, and marginal osteophyte formation, are present. (C) Right ankle-lateral view: calcification of the Achilles tendon and plantar fascia is seen. Degenerative changes are present in the talocalcaneal and calcaneocuboid (six men) aged 50-94. In seven of 10-20 years. In three patients the diagnosis was these eight patients examination of the joint Probable CPPD-CDD the diagnosis was made at the first attack of acute arthritis. 政 
Chronic CPPD-CDD with characteristic structural abnormalities of osteoarthritis (OA) was the presentation in five patients. Premature aggressive, destructive $\mathrm{OA}$ of the knee was seen in two patients. In both of them the disease started earlier than 50 years of age. Both required knee replacement within 10 years of the onset of symptoms. Severe generalised OA with deformities and disability, interspersed with acute intermittent monarthritis in some of the large joints in the arms and legs, was the presentation in two patients. In one, a woman, the first symptoms of an insidious onset of non-inflammatory polyarthritis affecting small and large joints in the arms and legs started at the age of 38. Interspersed with this were episodes of acute arthritis in some of the joints-namely, knee, wrists, elbows. Extensive chondrocalcinosis in most of the peripheral and axial joints, with features of aggressive OA in joints not usually affected in primary osteoarthritis-namely, wrists and metacarpophalangeal (MCP) joints, was demonstrable on radiological examination (fig $1 \mathrm{~A}-\mathrm{C}$ ). Synovial calcification was present in most of the MCP joints of both the hands and the first carpometacarpal joint on the left side, with capsular calcification of the second right proximal interphalangeal joint. No obvious underlying cause was detected on detailed investigations during her visit to the United Kingdom.

The second patient was a 75 year old Kuwaiti man who had had a renal transplant 10 years previously, with chronic pains and swellings in several peripheral large and small joints in the arms and legs, interspersed with acute attacks of fever, pain, and swelling in the knee joints since 1982, and in the elbow joints since 1997. He had been noted to be hyperuricaemic on several occasions in the past. Joint imaging showed advanced aggressive $\mathrm{OA}$ in the various large and small joints in the arms and legs, with chondrocalcinosis in the knee. Synovial fluid

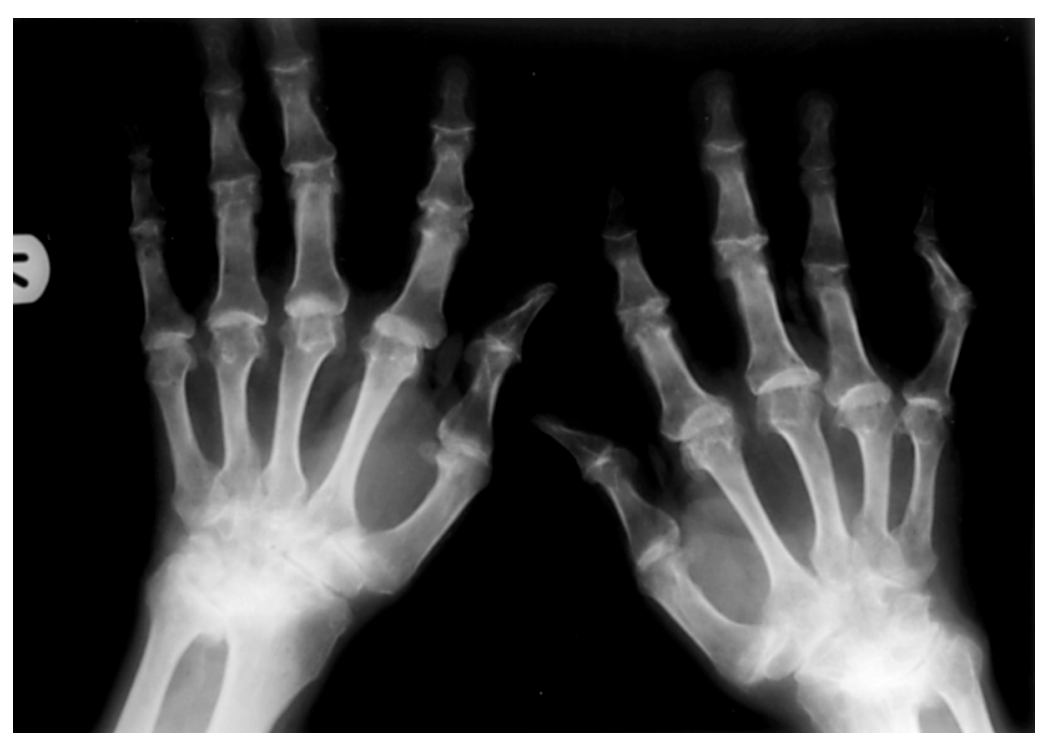

Figure 2 Radiographs of the wrists and hands: osteoarthritis of the distal interphalangeal, proximal interphalangeal, and metacarpophalangeal joints with calcium pyrophosphate
dihydrate deposition disease (CPPD-CDD). The hook-like drooping osteophytes on the radial aspects of the heads of the metacarpal joints and ulnar bones, destructive symmetrical bilateral arthritis of the wrists with scapholunate advanced collapse, radiocarpal joint space
narrowing, and subchondral sclerosis are all characteristic features of CPPDD-CDD. showed large numbers of CPPD crystals in association with crystals of monosodium urate. A 50 year old man had had RA-like extensive disease of small and large joints in his arms and legs, with ulnar deviation of the digits, since the ge of 35 years $\mathrm{He}$ also had low rheumatoid factor titres and high erythrocyte sedimentation rate and $\mathrm{C}$ reactive protein levels. However, the presence of typical hook-like drooping osteophytes on the radial aspects of the metacarpal heads with normal bone density (fig 2), other features of extensive aggressive A at several other joints usually not affected in $\mathrm{OA}$ (wrists, $\mathrm{MCP}$ joints), and syn in OA (wrists, MCP joints), and synovial fluid from the knee showing CPPD crystals, confirmed the diagnosis of CPPD-CDD. Haemochromatosis and other possible associated diseases were excluded clinically and by relevan investigations.

Acute arthritis of the knee was the presentation in three patients. Radiological examination showed typical destructive osteoarthritic changes with bilateral chondrocalcinosis in all of them. The synovial fluid was inflammatory and positive for CPPD crystals.

In five patients with probable CPPD-CDD the presentation was that of chondrocalcinosis with haemorrhagic acute knee arthritis. However, synovial fluid examination was negative for crystals as well as microbial cultures. An additional patient, a 75 year old man, had acute wrist arthritis with chondrocalcinosis of the knee. Aspirated fluid was negative for crystals. The microbial cultures were also negative. Al the patients responded satisfactorily to nonteroidal anti-inflammatory drugs with intraarticular corticosteroid injections.

A search for a possible underlying cause for the disease in young patients included investigations for hypothyroidism, hyperparathyroidism, haemochromatosis, hypomagnesaemia, and hypophosphatasia. One patient, in whom the disease started at age 36, was studied more extensively during her visit to the United Kingdom. However, no underlying cause could be established in any of these patients.

\section{Discussion}

This study showed that $2 \%$ of the patients presenting to rheumatology clinic with knee arthritis showed chondrocalcinosis. When the younger age of the group studied is taken into account this figure was comparable with those found in studies from the UK and USA. ${ }^{10}{ }^{11}$
Among crystal deposition diseases, CPPD$\mathrm{CDD}$ is the second most common disease after gout, ${ }^{1}$ but there are many fewer reports of this disease than of gout. A search for CPPD-CDD from Asia showed only one series from Thailand and a single case report from Qatar, in the Gulf Region. ${ }^{5} 6$ The reasons for this pauity of reports on CPPD-CDD are not obvious. city of reports on CPPD-CDD are not obvious.
Our study re-emphasised some of the main Our study re-emphasised some of the main disease does occur among Arabs and that its presentation is similar to that described from Western countries. ${ }^{1812}$ The study also confirmed the occurrence of this Arabs from North Africa, especially Tunisia, 
from where cases, case series, and familial cases have been described (cited by Reginato and Hamza $e t a l^{2}$ ).

Radiological changes in CPPD-CDD are characterised by features of aggressive destructive OA (massive osteophytes, subchondral bony sclerosis) in the affected joints. ${ }^{13}$ In addition, besides involvement of joints commonly affected in OA - namely, hip, knee, distal interphalangeal joint, the disease prominently affects joints not usually involved in primary OA, such as the wrists and MCP joints. ${ }^{14}$ Patients presented in this report showed these features, including aggressive destructive OA and OA affecting joints that would be considered unusual for primary OA

The main lessons learnt from this study were, firstly, that CPPD-CDD does occur among Middle-Eastern Arabs, possibly as frequently as reported from North Africa and Western countries. Secondly, this disease must be considered as a differential diagnosis of acute monarthritis in adults, especially if the knee is the affected joint and also among those presenting with arthritis resembling generalised primary OA but, at a relatively younger age, with the involvement of joints considered unusual for OA. Such patients must be carefully examined for chondrocalcinosis and CPPD crystals in the joint aspirates.

The authors thank Dr SM Karam, Associate Professor of
Anatomy, Faculty of Medicine, Kuwait University for his cooperation and help in the differential interference microscopy.
1 Rosenthal AK. Crystal arthropathies. In: Maddison P, Isen, Glass D, eds. Oxford textbook 1998:1555-81.

2 Reginato AJ, Reginato AM. Diseases associated with deposition of calcium pyrophosphate or hydroxyapatite. In ,

cium pyrophosphate crysta deposition disease; pseudogout; articular chondrocalcinosis. In: McCarty DJ, Koopman WJ, eds. Arthritis and allied 1993:1835-72.

Hamza $M$, Meddeb N, Bardin T. Hereditary chondrocalinosis in a Tunisian family. Clin Exp Rheumatol 1992;10 $43-9$.

5 Louthrenoo W, Sukitawut W. Calcium pyrophosphate dihydrate crystal deposition: a clinical and laboratory analys
of 91 Thai patients. J Med Assoc Thai 1999:82:569-76. 6 Hammoudeh MAU, Siam AR. Pseudogout in a young patient. Clin Rheumatol 1998;17:242-5.

Malaviya AN, Al-All N, Al-Gaurer S, Sharma P, Aluricemia among Kuwaitis possibly without proportione increase in gout. Br J Rheumatol 1997;36:405-6.

8 Ryan LM, McCarty DJ. Calcium pyrophosphate crystal deposition disease; pseudogout; articular chondrocalcinoed. Baltimore: Williams and Wilkins, 1997:2103-25. 13-

9 Schumacher RH Jr. Synovial fluid analysis and synovia biopsy. In: Kelly WN, Harris ED Jr, Ruddy S, Sledge CB, eds. Textbook of theumatology. 5 th ed. Phill

Felson DT, Anderson JJ, Naimark A, Kannel W, Meenan its association with knondrocalcinosis in the elderly and Study. J Rheumatol 1989;16:1241-5.

1 Jones AC, Chuck AI, Arie EA, Green DJ, Doherty M. Diseases associated with calcium pyrophosphate depositio ritis Rheum 1992;22:188-202. it under-recognized? Bull Rheum Dis 1995;44:3-5.

DS, Martel W. Radiologic m $m$ ifestations of the crysta14 Preidler KW, Brodssmann J Resnick D. Osteoarthritis. Semin Roentgenol 1996;31:208-19. 